\title{
Strong Governance, Trusted Leadership and Clear Communication: A Descriptive Narrative of an Australian Public Hospital Response to COVID-19
}

\author{
Katherine Maka, Graeme Loy, Amanda Green, Tiffany Sly, Abbey Roach \\ Western Sydney Local Health District: COVID-19 Response, Westmead, Australia \\ Email: katherine.maka@health.nsw.gov.au
}

How to cite this paper: Maka, K., Loy, G. Green, A., Sly, T., \& Roach, A. (2021). Strong Governance, Trusted Leadership and Clear Communication: A Descriptive Narrative of an Australian Public Hospital Response to COVID-19. Journal of Service Science and Management, 14, 291-304. https://doi.org/10.4236/jssm.2021.143018

Received: April 16, 2021

Accepted: June 4, 2021

Published: June 7, 2021

Copyright $\odot 2021$ by author(s) and Scientific Research Publishing Inc. This work is licensed under the Creative Commons Attribution International License (CC BY 4.0).

http://creativecommons.org/licenses/by/4.0/ Open Access

\begin{abstract}
This paper provides a critical reflection of an Australian healthcare organisation's approach to managing the impact of the COVID-19 pandemic. A strong focus is placed on the importance of both staff and patient mental health and wellbeing which is underpinned by clear governance, communication and leadership. The challenges of working in uncertainty are widely acknowledged and documented, and operating as a health service provider during this time surfaced many unknowns. This paper seeks to improve our understanding of the influence of governance structures, communication and leadership when functioning in uncertain times. Key learnings that may be considered by other multifaceted organisations include; governance structures that recognise the importance of involving subject matter experts to reinforce organisational trust in hierarchical decision making, establishing systems that facilitate structured and informed decision making that is supported by visible communications, valuing agility and responsiveness in the workforce through shared listening and information sharing, and consistent front and centre messaging of organisational priorities to reinforce the natural flow of human behaviour change.
\end{abstract}

\section{Keywords}

COVID-19, Governance, Trust, Leadership, Communication

\section{Introduction}

Western Sydney Local Health District (WSLHD) provides healthcare to more than 946,000 residents in Sydney's west as well as speciality services across New 
South Wales. Healthcare is delivered to more than 120 suburbs, which brings a diversity of populations, cultures, economic, social and healthcare needs. Delivery of healthcare is supported by over 13,000 staff, across a number of campuses including Westmead, Auburn, Cumberland, Blacktown and Mt Druitt hospitals as well as a network of comprehensive integrated health and community based services. There is expertise and capacity across the district to provide tertiary healthcare services, meaning that complex health needs are addressed across WSLHD's facilities, for community care needs.

As the designated high-consequence infectious diseases facility in NSW, Westmead Hospital had the right infrastructure to support the treatment and management of COVID-19 patients, with expert staff, appropriate isolation rooms, infection prevention and control measures, education and training programs, and access to personal protective equipment (High Consequence Infectious Diseases, n.d.). As COVID-19 cases grew internationally, Australia did not see the same rapid spread of the pandemic. Despite this, WSLHD recognised a level of urgency to consider leadership and governance structures prior to the arrival of the first COVID-19 patient. This would provide the foundation to proactively consider and respond to staff, patients and wider community needs.

On 25 January 2020, Westmead Hospital received Australia's first case of COVID-19 (Shaban et al., 2021). Responses to healthcare disasters and emergencies traditionally involve activation of an emergency operations centre which shifts leadership and decision making away from usual practices to a targeted emergency management team for typically short term issues management (Mawardi et al., 2020). At the emergence of COVID-19 in Australia, each Local Health District stood up emergency operations centres which aligned with NSW Health's overarching emergency response centres, being the State Health Emergency Operations Centre (SHEOC), and the Public Health Emergency Operations Centre (PHEOC).

Despite the command and control style of leadership that emergency operations management brings, WSLHD's Executive team quickly realised the necessity for agile, responsive leadership and decision-making in the midst of the longevity of the pandemic. As such, WSLHD moved away from the emergency operations centre governance model, and pivoted existing governance structures to lead a COVID-19 response, facilitating consistent, clear, structured decision-making from familiar organisational leadership positions. This was integral for WSLHD to be able to maintain usual healthcare and service delivery for the non-COVID-19 community whilst managing the uncertainty and complexities of the pandemic.

WSLHD witnessed many key outcomes during the first year of the COVID-19 pandemic in Australia, including establishing an effective governance structure and supporting systems such as communication, monitoring service provision during the pandemic, ensuring access to personal protective equipment (PPE), pivoting education and training models, understanding the importance of men- 
tal health and wellbeing resulting in workforce responsiveness, and developing COVID-19 models of care (Kam et al., 2021). These key outcomes will be discussed in this paper under eight sub-headings.

\section{Key Outcomes}

\subsection{WSLHD's COVID-19 Governance Structure}

With the advent of the pandemic, unprecedented in scale, it was clear WSLHD needed a novel approach to leadership and governance in order to be adaptable and resilient (Pearce et al., 2020). The existing district governance structure, consisting of broad clinical and corporate management portfolios, and headed by the Chief Executive, already provided the right scaffolding for decision-making and managing uncertainty. Research acknowledges that "large-scale human behaviour change" can be achieved through the confidence of existing trusted leadership (Ahern \& Loh, 2020: p. 1). By bringing forward the established and trusted leadership and governance structures, WSLHD was be able respond to the calling of the COVID-19 pandemic through dynamic human and operational change management.

New forums were quickly established to facilitate responsive and timely decision-making as COVID-19 cases grew in Australia. These forums consisted of a daily district and facility executive huddle focused on operational facility management. As opposed to the traditional process of decision making through preparation of a briefing paper with a series of hierarchical approvals, decisions were raised and discussed with senior leaders, minuted and actioned on a real time basis, daily.

A weekly COVID-19 District Executive Committee meeting with a strong clinical focus was also introduced to facilitate strategic decision-making around WSLHD's pandemic management. Strong engagement with key community stakeholders, including Western Sydney's Primary Health Network, and key external providers, as well as key WSLHD clinical leaders were in place. An additional layer of clinical advice provided through a COVID-19 Clinical Expert Advisory Group, which was established to facilitate the mitigation of clinical and organisational risks associated with the COVID-19 pandemic. This group brought together clinical leads across different medical disciplines to provide a forum for regular multidisciplinary consideration of COVID-19 in Western Sydney, identifying and monitoring clinical risks and concerns for escalation and facilitating interdepartmental information sharing. The daily district and facility Executive Huddle, weekly COVID-19 District Executive Committee meeting and the COVID-19 Clinical Expert Advisory Groups were the corner stone of transparent, collegial, agile and visible governance across the district, and were quickly integrated and accepted as the key decision-making engines for all COVID-19 matters.

To facilitate a coordinated approach that would have stringent oversight on all local and relevant NSW Ministry of Health COVID-19 matters, a COVID-19 
Response Team was formed. This team had a direct report to WSLHD's Chief Executive, however did not sit as part of the District's executive structure. This focussed the team to be accessible, dynamic and responsive to the district's COVID-19 needs, and act as a centralised information repository which received large volumes of incoming and outgoing correspondence involving the Ministry of Health and other regulatory bodies. The roles and responsibilities of this team were designated to mitigate potential errors from any lack of coordination caused by the speed of incoming correspondence that required healthcare facilities to continuously pivot to respond to the latest pandemic advice. This provided a single internal and external contact point for all WSLHD COVID-19 related matters, ensured that decision-making was pushed through WSLHD's established COVID-19 governance structures, and facilitated informed decision-making across all management levels throughout WSLHD.

Centralised reporting and recording of COVID-19 related correspondence was driven by internal corporate records management experts, resulting in information transparency and accessibility across the District. This facilitated a vigorous timeframe management process and further strengthened WSLHD's coordinated response to COVID-19 demands. It assisted WSLHD to meet State and Federal requirements and deadlines, as well as monitoring internal project progressions. Internal corporate records management systems were used, allowing WSLHD to utilise existing corporate knowledge and pivot existing skill sets to ensure accuracy and attention to detail. This was quickly regarded as the single source of reliable information across WSLHD, during a time of rapidly changing advice being received from multiple sources. This aided WSLHD executive in maintaining robust oversight over all matters related to the pandemic, as well as fostering staff confidence in WSLHD's pandemic response management by ensuring that communications contained current and relevant information.

\subsection{Communication}

There is a stream of evidence that confirms the importance of communication during uncertain times (Grace \& Tham, 2021). Sound communication is vital and is inclusive of healthcare settings (Wu \& John, 2021). COVID-19 set the stage for a strong focus on communication. There was a need to provide clear and concise COVID-19 messaging without causing fallacies about the status of COVID-19 in Australia. Effective communication across WSLHD was achieved through a daily Chief Executive memo that was sent to all staff via email. This outlined current community COVID-19 status, upcoming changes to prepare for, and reinforced expected behaviours. Each daily correspondence was also posted on WSLHD's purpose-built staff website to increase accessibility and create an inclusive information archive. This communication medium was a platform that built staff compliance in engaging in correct and safe practices and reduced unnecessary anxieties through staff feeling informed (Argenti, 2020). Required workplace changes were communicated via the memo, building as- 
surance and transparency in this information sharing platform. Such communication has been recognised as paramount particularly when working within a large organisation (Argenti, 2020).

Risk communication and community engagement (World Health Organization, 2020) are acknowledged as being highly important during a pandemic to support effective communication. This was demonstrated through ensuring that COVID-19 safe messages were accessible and relevant to meet the demands of changing COVID-19 risks across various local community groups. Research highlights that strong communication from trusted sources reduces fear caused by conflicting information (Hyland-Wood et al., 2021). WSLHD capitalised on internal communication expertise, as well as clinician and community links across Western Sydney to address different community group communication needs. Trust nurtured the need to grow relationships with community leaders to swiftly address COVID-19 risks and concerns (Maher \& Murphet, 2020). Appropriate funnelling of communication across staff and community cohorts contributed greatly to fostering trust. This was particularly important to the continued growth of staff confidence in WSLHD's pandemic governance and management strategies.

The success of WSLHD's communication during the height of the pandemic was reinforced by results from a survey led by the district's Workplace Wellness team in late 2020. Of particular note is when staff were asked if "senior managers kept staff well informed about what was going on during COVID-19", 67\% of WSLHD's staff respondents answered positively. This was a significant increase to results from a similar question in NSW Health's 2019 Pulse - My Experience Matters Survey (39\% positive). This also saw a positive shift in staff's feelings of confidence in senior management's ability to manage change, seeing a $14 \%$ increase in 2020 results compared to that of 2019.

\subsection{Monitoring Service Provision during Pandemic}

At the onset of the pandemic, high uncertainty was guaranteed because of a lack of solid knowledge about the actual impact of COVID-19 within NSW (Koffman et al., 2020). With emerging COVID-19 cases in NSW and the introduction of state-wide community restrictions, there was a notable level of fear across patient and staff communities in regard to accessing onsite healthcare services (Fisher et al., 2020). This saw changes in patient attendance patterns across both inpatient and outpatient settings (Kam et al., 2020; Sutherland et al., 2020). Research has suggested the importance of monitoring patient flow and presentations to healthcare setting (Lazzerini et al., 2020). WSLHD were able to ensure that there was visibility over service provision during COVID-19 through a service suspension, recommencement and amendment process. This supported shared clinical decision making methodology and transparency for service provision changes (Abrams et al., 2020).

This service suspension, recommencement and amendment process encour- 
aged clinician and executive thought processes that were focussed on meeting the healthcare needs of WSLHD's community, together with a strong focus on COVID-19 safe service delivery and a shared assessment of associated risk. This was overseen by stringent governance through established COVID-19 executive forums, which enabled many services to remain operational during the pandemic, with service delivery that aligned with the latest COVID-19 safety advice. This process highlighted opportunities to trial alternate models of care, as evidenced by the establishment of telehealth service delivery across a range of patient settings. These changes to usual care practices would not have been explored without the necessity to operate in a pandemic response environment, which fostered significant clinical engagement in exploring alternate modes of healthcare delivery.

\subsection{Access to Personal Protective Equipment (PPE)}

The use of appropriately applied PPE to prevent the transmission of COVID-19 and to protect health care workers is essential (Kam et al., 2021; Livingston et al., 2020; Ranney et al., 2020). Access to PPE among healthcare workers has also been shown to reduce additional stress, supporting employee's satisfaction (Zhang et al., 2020). With increasingly limited prevalence of PPE stock in the national medical stockpile, and global supply chain challenges, the need for PPE monitoring, centralisation and management to allow for contingency planning was recognised by WSLHD (Cook, 2020; Kam et al., 2021; Rowan \& Laffey, 2020).

"The 'PPE Predict' tool was developed by WSLHD and University of Sydney staff to track equipment stock levels, and predict PPE burn rate, that is, how quickly the stock will be used based on the required type for patients in the hospital (Kam et al., 2021; Raja et al., 2020). This tool was instrumental in maximising optimal use of PPE, monitoring current and future PPE stock levels, limiting inappropriate use or wastage of PPE, and providing WSLHD management with a clear monitoring system for fast escalation of PPE stock concerns, which could then be addressed at a state-wide level if required (Cook, 2020; Zhang et al., 2021). This tool has ensured that WSLHD can continually meet staff and patient PPE demand, which is paramount to ongoing health service delivery to western Sydney and therefore ensuring the safety of WSLHD's frontline healthcare workers (Kam et al., 2021; Raja et al., 2020).

\subsection{Education and Training}

As international evidence emerged to inform healthcare facilities around their COVID-19 management, WSLHD realised the need to deliver alternate modes of education and training to support the ongoing connectedness of students, staff and peers, to build workforce capacity, and to develop best practice strategies (Dost et al., 2020). Emerging COVID-19 restrictions across Australia resulted in a diminished capacity to provide face to face education opportunities 
due to restrictions such as physical distancing (Bell et al., 2020). As a large healthcare teaching district, WSLHD were invested in continuing to provide education and training to staff to ensure workplace readiness and responsiveness.

A key focus for WSLHD's education and training was staff preparedness for delivering care to COVID-19 patients. Early experiences and data from Europe have revealed higher numbers of healthcare workers with COVID-19, with inadequate PPE usage as one contributing factor to the spread among healthcare workers (Ehrlich et al., 2020). To combat this, the literature recognises the need for rigorous training standards in order to ensure correct and appropriate PPE usage (Kumar et al., 2020). Simulation Labs are a proven way to "improve safety orientated behaviours" which have a direct positive impact on patient safety outcomes (Ingrassia et al., 2020). WSLHD developed simulation based training to deliver education on appropriate PPE usage and intensive care specific skills training. Simulation training was also used to upskill existing staff, resulting in additional workforce capacity to respond to fluctuating healthcare demands in a pandemic environment.

Further to simulation training, WSLHD moved classroom-based training courses to an online platform. This was especially important for the transition of the mandatory workplace orientation course. This course was transitioned from wholly classroom based to online delivery in three days, and meant that over 150 new recruits could continue to enter WSLHD despite the pandemic environment and experience facilitated workplace readiness. WSLHD's success in pivoting education and training delivery was due to the availability of information communication technologies along with onsite expertise in health worker education delivery (Allen et al., 2020).

These modes of education delivery are able to be tracked, and WSLHD capitalised on the availability of completed course information to ensure essential staff cohorts were receiving and attending training. This also facilitated training and course content feedback between educator and student, allowing education to be tailored to staff needs. These combined provided surety that WSLHD staff was well equipped to translate their learnings into their area of work. No WSLHD staff member acquired COVID-19 through the workplace despite multiple community outbreaks. This suggests anecdotally that the training delivered to WSLHD staff was a key mechanism to negate the potential risks when working as a healthcare worker during a pandemic.

\subsection{Mental Health and Wellbeing}

Rapidly changing environments, often associated with a level of uncertainty, can influence an individual's anxiety, distress and mental health (Ehrlich et al., 2020; Xiong et al., 2020). Levels of stress can also influence mental health issues across many populations and have been shown to be a direct result of the uncertainty and change that COVID-19 delivered (Wang et al., 2021; Wang et al., 2020). WSLHD utilised existing resources by way of expertise and engagement of the 
Staff Wellness Team, driving the establishment of a targeted COVID-19 Staff Wellness strategy. This included a COVID-19 assist line for staff, as well as drop-in centres, virtual wellness checks, and mindfulness and mediation sessions. Coaching sessions were also provided to managers with a strong focus on education in managing change and staff stress. Despite the uncertainty of a pandemic environment, anecdotal data suggests that WSLHD staff felt well supported in their work environments during this time. These strategies were impactful in managing stress and self-perceptions of anxiety and depression as well as improving feelings of wellbeing, enabling WSLHD's workforce to maintain productivity and provide healthcare services to the Western Sydney community (Behan, 2020).

Early in the midst of the pandemic, NSW Health recognised the potential impact that being in hotel quarantine may have on people's mental health. To address this need, WSLHD designed and implemented a COVID-19 Mental Health Response Team to provide multidisciplinary services and support to all quarantined travellers residing in Sydney hotels. This team comprised of Clinical Nurse Consultants, Registered Nurses, Social Workers, Occupational Therapists, Psychologists and Psychiatrists, and provided intervention, counselling and support. Knowing that psychological distress associated with isolation can be supported through human connectedness (Conversano et al., 2020), WSLHD's Mental Health Response Team showed strong outcomes in reducing levels of stress (Brooks et al., 2020). Most people were managed via telehealth service delivery, however site visits were conducted when recognised as the most appropriate service for the traveller's care needs. Given cultural diversity and vulnerable persons amongst returned travellers in Sydney's quarantine hotels, a responsive strategy was adopted utilising health care interpreter services and multi-cultural healthcare workers in order to deliver appropriate and sensitive support (Brooks et al., 2020).

These wellbeing and mental health strategies were made visible through regular reporting to the daily executive covid-19 meeting, reinforcing the importance of strong governance in this space. This enabled agility in troubleshooting, addressed new learnings, and facilitated the breakdown of silos to reinforce the importance of holistic wellness in the workplace and the community (Rajam et al., 2020).

\subsection{Workforce Responsiveness}

Forward planning in all aspects of health service delivery has been attributed as a catalyst that aids a level of preparedness across the healthcare system (Leite et al., 2020). WSLHD remained proactive in ensuring that they would be able to continue to use their workforce to respond to the changing healthcare landscape driven by the COVID-19 pandemic in NSW. This was in the face of needing to support the state effort, where experienced staff was required to support NSW Health's SHEOC. Through recognising a need to avoid local staffing shortages 
and potential service disruption, WSLHD proactively planned contingency staffing to respond to COVID-19 surges across the state. WSLHD staff with appropriate capability and capacity to support NSW COVID-19 response strategies was identified for allocation by local facility management, demonstrating forward thinking and allowing smooth transition of staff across the NSW Health's clinical workforce. This process became the mainstay to ensure that daily local clinical staffing rosters were not disrupted by state surge staffing requirements, especially during times of peak COVID-19 activity in Western Sydney. This also ensured that the healthcare needs of non-COVID-19 patients in Western Sydney were met (Leite et al., 2020).

With growing uncertainty associated with COVID-19, adaptability, flexibility and agility in WSLHD's responsiveness was essential to address the forced workforce changes. For example, as seen in the necessary temporary shutdown of all Oral Health Services across WSLHD at the onset of the pandemic in Australia. Staffs were quickly moved into new COVID-19 specific service roles that were required across WSLHD facilities, such as facility entry screeners. Individuals dealing with change or uncertainty are able to respond positively when provided with the right platforms of leadership, such as open communication, role clarity and flows of work (Suresh et al., 2021). This was evidenced by the mobilisation of Oral Health staff out of their usual clinical space during their temporary shutdown. Strong governance reinforced clear reporting lines, resulting in the dynamic redeployment of this staffing cohort to meet emerging frontline COVID-19 requirements. This response was further strengthened by WSLHD's COVID-19 focussed communication strategies, allowing additional staff reassignments and targeted new starter recruitment to facilitate a strong COVID-19 response across WSLHD (Bielicki et al., 2020).

Furthermore, centralised governance structures within WSLHD reinforced the collective leadership approach to decision-making across the district as a whole rather than problem solving at the facility or health service level. This was the impetus in identifying possible pressure points where risk mitigation proactively supported preparedness. For example, due to known risks of COVID-19 in Western Sydney's aged care population, WSLHD stood up a temporary Residential Aged Care Taskforce in an unprecedented response to proactively address these risks (Cousins, 2020). In addition, the collective leadership approach fostered flexibility in staff utilisation, such as using disability services for mask packing, job opportunities for Western Sydney's Aboriginal and Torres Strait Islander population and administration staff to conduct facility entry screening, and upskilling physiotherapists for intensive care.

\subsection{COVID-19 Hospital and Models of Care}

As global healthcare provision crises emerged in the grip of the pandemic, countries were faced with many challenges such as reduced workforce capacity, decreased access to appropriate equipment and diminished hospital capacity to 
treat all patients requiring inpatient care (Meyer et al., 2020). The ability to care for COVID-19 patients away from non-infected patients has been recognised as an optimal environment for reducing the spread of the virus to other patients and staff, and provides opportunity to build strong clinical capabilities in staff (Meyer et al., 2020). WSLHD were in a unique position to plan a proactive COVID-19 response due to access to an empty, yet complete, brand new clinical building on the campus of the Westmead Health Precinct. The ability to stand up an isolated COVID-19 dedicated hospital provided the opportunity to develop an extensive operational plan. Research has acknowledged the value of proactive strategic planning to pre-empt the trajectory of outcomes caused by a pandemic (Sharma et al., 2021). Due to the lack of COVID-19 prevalence in Australia there was no urgent need to operationalise the COVID-19 Hospital (Scott et al., 2020).

Nevertheless, planning produced detailed governance and workforce structures, and evidenced based COVID-19 models of care for patient care in Intensive Care Units, Emergency Departments and ward based settings. Capitalising on strong clinical stewardship, best practice and evidence based models of care were developed. Empowered by extensive clinical knowledge and experience, these plans were able to be operationalised and translated for use across existing clinical building settings.

Low case numbers saw the management of patients within Westmead Hospital and in the community which allowed the developed models of care to be utilised and reformed to meet the emerging needs of COVID-19. This highlighted that continuous learning from practical experience is critical and that dynamic and adaptable models of care are essential in order to meet healthcare needs and patient demand (Ahern \& Loh, 2020; Kumaraiah et al., 2020).

\section{Conclusion}

This paper provides a high level overview of a healthcare organisation's key strategies and their associated outcomes when responding to the COVID-19 pandemic in Australia. One of the limitations of this paper is that this does not provide qualitative and quantitative outcome measures to substantiate the benefits of the key outcomes which have been identified. WSLHD's ability to navigate the uncertain waters of the COVID-19 pandemic has been strongly attributed to clear governance systems and structures that are founded on sound and trusted leadership which harnessed key clinical relationships. Underpinning this has been consistent, transparent communication channels that have reached all levels of staff, from frontline clinicians, to middle managers, to senior executive, and across Western Sydney's local communities. Strong service provision monitoring ensures access to essential clinical services, reassuring staff that they can deliver services safely, and more importantly reassuring patients that they can access necessary healthcare. The monitoring afforded to WSLHD's clinical streams fosters and spreads innovative thinking, resulting in a significant uptake 
of telehealth models of care to ensure continuation of service delivery. Through shifting traditional educational delivery modes, sound learning outcomes are maintained particularly in the area of PPE use. Investing in the mental health and wellbeing of staff was evidenced in building trust in leadership, resulting in a workforce that is adaptable and receptive to change. This is essential to adapt current models of care and address community needs, such as meeting the unique healthcare requirements of COVID-19 patients. Given the successful ability of WSLHD to adjust during times of uncertainty, it will be important to draw on the key learnings of strong governance, trusted leadership and clear communication in order to continue to meet the ever changing landscape of COVID-19.

\section{Conflicts of Interest}

The authors declare no conflicts of interest regarding the publication of this paper.

\section{References}

Abrams, E. M., Shaker, M., Oppenheimer, J., Davis, R. S., Bukstein, D. A., \& Greenhawt, M. (2020). The Challenges and Opportunities for Shared Decision Making Highlighted by COVID-19. The Journal of Allergy AND Clinical Immunology: In Practice, 8, 2474-2480. https://doi.org/10.1016/j.jaip.2020.07.003

Ahern, S., \& Loh, E. (2020). Leadership during the COVID-19 Pandemic: Building and Sustaining Trust in Times of Uncertainty. BMJ Leader, 1-4.

https://doi.org/10.1136/leader-2020-000271

Allen, J., Rowan, L., \& Singh, P. (2020). Teaching and Teacher Education in the Time of COVID-19. Asia-Pacific Journal of Teacher Education, 48, 233-236. https://doi.org/10.1080/1359866X.2020.1752051

Argenti, P. (2020). Communicating through the Coronavirus Crisis. https://hbr.org/2020/03/communicating-through-the-coronavirus-crisis

Behan, C. (2020). The Benefits of Meditation and Mindfulness Practices during Times of Crisis Such as COVID-19. Irish Journal of Psychological Medicine, 37, 256-258. https://doi.org/10.1017/ipm.2020.38

Bell, D. J., Self, M. M., Davis, C., Conway, F., Washburn, J. J., \& Crepeau-Hobson, F. (2020). Health Service Psychology Education and Training in the Time of COVID-19: Challenges and Opportunities. American Psychology, 75, 919-932. https://doi.org/10.1037/amp0000673

Bielicki, J. A., Duval, X., Gobat, N., Goossens, H., Koopmans, M., Tacconelli, E., \& van der Werf, S. (2020). Monitoring Approaches for Health-Care Workers during the COVID-19 Pandemic. Lancet Infectious Diseases, 20, 261-267. https://doi.org/10.1016/S1473-3099(20)30458-8

Brooks, S. K., Webster, R. K., Smith, L. E., Woodland, L., Wessely, S., Greenberg, N., \& Rubin, G. J. (2020). The Psychological Impact of Quarantine and How to Reduce It: Rapid Review of the Evidence. The Lancet, 395, 912-920. https://doi.org/10.1016/S0140-6736(20)30460-8

Clinical Excellence Commission (n.d.). High Consequence Infectious Diseases. https://www.cec.health.nsw.gov.au/keep-patients-safe/infection-prevention-and-contro 1/high-consequence-infectious-diseases 
Conversano, C., Di Giuseppe, M., Miccoli, M., Ciacchini, R., Gemignani, A., \& Orrù, G. (2020). Mindfulness, Age and Gender as Protective Factors against Psychological Distress during COVID-19 Pandemic. Frontiers in Psychology, 11, 1900.

https://doi.org/10.3389/fpsyg.2020.01900

Cook, T. M. (2020). Personal Protective Equipment during the Coronavirus Disease (COVID). 2019 Pandemic-A Narrative Review. Anaesthesia, 75, 920-927. https://doi.org/10.1111/anae.15071

Cousins, S. (2020). Experts Criticise Australia's Aged Care Failings over COVID-19. The Lancet, 396, 1322-1323. https://doi.org/10.1016/S0140-6736(20)32206-6

Dost, S., Hossain, A., Shehab, M., Abdelwahed, A., \& Al-Nusair, L. (2020). Perceptions of Medical Students towards Online Teaching during the COVID-19 Pandemic: A National Cross-Sectional Survey of 2721 UK Medical Students. British Medical Journal Open, 10, e042378. https://doi.org/10.1136/bmjopen-2020-042378

Ehrlich, H., McKenney, M., \& Elkbuli, A. (2020). Protecting Our Healthcare Workers during the COVID-19 Pandemic. Correspondence/American Journal of Emergency Medicine, 38, 1515-1539. https://doi.org/10.1016/j.ajem.2020.04.024

Fisher, J. R. W., Tran, T. D., Hammarberg, K., Sastry, J., Nguyen, H., Rowe, H., Popplestone, S., Stocker, R., Stubber, C., \& Kirkman, M. (2020). Mental Health of People in Australia in the First Month of COVID-19 Restrictions: A National Survey. Medical Journal of Australia, 213, 458-464. https://doi.org/10.5694/mja2.50831

Grace, R., \& Tham, J. C. K. (2021). Adapting Uncertainty Reduction Theory for Crisis Communication: Guidelines for Technical Communicators. Journal of Business and Technical Communication, 35, 110-117. https://doi.org/10.1177/1050651920959188

Hyland-Wood, B., Gardner, J., Leask, J., \& Ecker, U. K. H. (2021). Toward Effective Government Communication Strategies in the Era of COVID-19. Humanities and Social Sciences Communications, 8, 30. https://doi.org/10.1057/s41599-020-00701-w

Ingrassia, P. L., Capogna, G., Diaz-Navarro, C., Szyld, D., Tomola, S., \& Leon-Castelao, E. (2020). COVID-19 Crisis, Safe Reopening of Simulation Centres and the New Normal: Food for Thought. BioMed Central Advances in Simulation, 5, 1-14.

https://doi.org/10.1186/s41077-020-00131-3

Kam, A. W., Chaudhry, S. G., Gunasekaran, N., White, A. J. R., Vukasovic, M., \& Fung, A. T. (2020). Fewer Presentations to Metropolitan Emergency Departments during the COVID-19 Pandemic. The Medical Journal of Australia, 213, 370-371. https://doi.org/10.5694/mja2.50769

Kam, A. W., King, N., Sharma, A., Philips, N., Nayyar, V., \& Shaban, R. Z. (2021). Personal Protective Equipment for the Care of Suspected and Confirmed COVID-19 Patients-Modelling Requirements and Burn Rate. Infection, Disease \& Health. https://doi.org/10.1016/j.idh.2021.04.001

Koffman, J., Gross, J., Etkind, S. N., \& Selman, L. (2020). Uncertainty and COVID-19: How Are We to Respond? Journal of the Royal Society of Medicine, 113, 211-216. https://doi.org/10.1177/0141076820930665

Kumar, J., Katto, M., Siddiqui, A. A., Sahito, B., Jamil, M., Rasheed, N., \& Ali, M. (2020). Knowledge, Attitude, and Practices of Healthcare Workers Regarding the Use of Face Mask to Limit the Spread of the New Coronavirus Disease (COVID-19). Cureus, 12, e7737. https://doi.org/10.7759/cureus.7737

Kumaraiah, D., Yip, N., \& Hill, L. (2020). Innovation ICU Physician Care Models: Covid-19 Pandemic at New York-Presbyterian. New England Journal of Medicine Catalyst, 1-6.

Lazzerini, M., Bardi, E., Apicella, A., Marchetti, F., Cardinale, F., \& Trobia, G. (2020). 
Delayed Access or Provision of Care in Italy Resulting from Fear of COVID-19. The Lancet, 4, e10-e11. https://doi.org/10.1016/S2352-4642(20)30108-5

Leite, H., Lindsay, C., \& Kumar, M. (2020). COVID-19 Outbreak: Implications on Healthcare Operations. The TQM Journal, 33, 247-256. https://doi.org/10.1108/TQM-05-2020-0111

Livingston, E., Desai, A., \& Berkwits, M. (2020). Sourcing Personal Protective Equipment during the COVID-19 Pandemic. JAMA, 323, 1912-1914. https://doi.org/10.1001/jama.2020.5317

Maher, R., \& Murphet, B. (2020). Community Engagement in Australia's COVID-19 Communications Response: Learning Lessons from the Humanitarian Sector. Media International Australia, 177, 113-118. https://doi.org/10.1177/1329878X20948289

Mawardi, F., Lestari, A. S., Randita, A. B. T., Kambey, D. R., \& Prijambada, I. D. (2020). Strengthening Primary Health Care: Emergency and Disaster Preparedness in Community with Multidisciplinary Approach (pp. 1-2). Society for Disaster Medicine and Public Health. https://doi.org/10.1017/dmp.2020.143

Meyer, G. S., Blanchfield, B. B., Bohmer, R. M. J., Mountford, J., \& Vanderwagen, C. (2020). Alternative Care Sites for the Covid-19 Pandemic: The Early U.S. and U.K. Experience. New England Journal of Medicine Catalyst, 1-6.

Pearce, A. P., Naumann, D. N., \& O’Reilly, D. (2020). Mission Command: Applying Principles of Military Leadership to the SARS-CoV-2 (COVID-19) Crisis. British Medical Journal Military Health, 167, 3-4. https://doi.org/10.1136/bmjmilitary-2020-001485

Raja, S., Patolia, H. H., \& Baffoe-Bonnie, A. W. (2020). Calculating an Institutional Personal Protective Equipment (PPE) Burn Rate to Project Future Usage Patterns during the 2020 COVID-19 Pandemic. Infection Control \& Hospital Epidemiology, 41, 1474-1475. https://doi.org/10.1017/ice.2020.190

Rajam, D., Koch, K., Rohrer, K., Bajnoczki, C., Socha, A., Voss, M., Nicod, M., Ridde, V., \& Koonin, J. (2020). Governance of the Covid-19 Response: A Call for More Inclusive and Transparent Decision-Making. BMJ Global Health, 5, e002655. https://doi.org/10.1136/bmjgh-2020-002655

Ranney, M. L., Griffeth, V., \& Jha, A. K. (2020). Critical Supply Shortages-The Need for Ventilators and Personal Protective Equipment during the Covid-19 Pandemic. The New England Journal of Medicine, 382, e41. https://doi.org/10.1056/NEJMp2006141

Rowan, N. J., \& Laffey, J. G. (2020). Challenges and Solutions for Addressing Critical Shortage of Supply Chain for Personal and Protective Equipment (PPE) Arising from Coronavirus Disease (COVID19) Pandemic-Case Study from the Republic of Ireland. Science of the Total Environment, 725, Article ID: 138532.

https://doi.org/10.1016/j.scitotenv.2020.138532

Scott, N., Palmer, A., Delport, D., Abeysuriya, R., Stuart, R., Kerr, C. C., Mistry, D., Klein, D., Sacks-Davis, R., Heath, K., Hainsworth, S., Pedrana, A., Stoove, M., Wilson, D., \& Hellard, M. E. (2020). Modelling the Impact of Reducing Control Measuring on the COVID-19 Pandemic in a Low Transmission Setting. The Medical Journal of Australia, 1-55. https://doi.org/10.1101/2020.06.11.20127027

Shaban, R. Z., Li, C., O’Sullivan, M. V. N., Gerrard, J., Stuart, R. L., Teh, J., Gilroy, N., Sorrell, T. C., White, E., Bag, S., Hackett, K., Chen, S. C. A., Kok, J., Dwyer, D. E., Iredell, J. R., Maddocks, S., Ferguson, P., Varshney, K., Carter, I., Barratt, R., Shaw, D. et al. (2021). COVID-19 in Australia: Our National Response to the First Cases of SARS-CoV-2 Infection during the Early Biocontainment Phase. Internal Medicine Journal, 51, 42-51. https://doi.org/10.1111/imj.15105

Sharma, A., Borah, S. B., \& Moses, A. C. (2021). Responses to COVID-19: The Role of 
Governance, Healthcare Infrastructure, and Learning from Pandemics. Journal of Business Research, 122, 597-607. https://doi.org/10.1016/j.jbusres.2020.09.011

Suresh, M., Roobaswathiny, A., \& Lakshmi Priyadarsini, S. (2021). A Study on the Factors That Influence the Agility of COVID-19 Hospitals. International Journal of Healthcare Management, 14, 290-299. https://doi.org/10.1080/20479700.2020.1870355

Sutherland, K., Chessman, J., Zhaob, J., Sarac, G., Shetty, A., Smith, S., Went, A., Dyson, S., \& Levesque, J. F. (2020). Impact of COVID-19 on Healthcare Activity in NSW, Australia. Public Health Research and Practice, 30, e3042030.

https://doi.org/10.17061/phrp3042030

Wang, H., Xia, Q., Xiong, Z., Li, Z., Xiang, W., Yuan, Y. et al. (2020). The Psychological Distress and Coping Styles in the Early Stages of the 2019 Coronavirus Disease (COVID-19). Epidemic in the General Mainland Chinese Population: A Web-Based Survey. PLoS ONE, 15, e0233410. https://doi.org/10.1371/journal.pone.0233410

Wang, Y., Di, Y., Ye, J., \& Wei, W. (2021). Study on the Public Psychological States and Its Related Factors during the Outbreak of Coronavirus Disease 2019 (COVID-19) in Some Regions of China. Psychology, Health \& Medicine, 26, 13-22. https://doi.org/10.1080/13548506.2020.1746817

World Health Organization (2020). Risk Communication and Community Engagement Readiness and Response to Coronavirus Disease (COVID-19): Interim Guidance. https://www.who.int/publications/i/item/risk-communication-and-community-engage ment-readiness-and-initial-response-for-novel-coronaviruses

Wu, J. H., \& John, S. D. (2021). The Ethics of COVID-19 Risk Communication (pp. 1-2). Society of General Internal Medicine. https://doi.org/10.1007/s11606-021-06600-3

Xiong, J., Lipsitz, O., Nasri, F., Lui, L. M. W., Gill, H., Phan, L., Chen-Li, D., Iacobucci, M., Ho, R., Majeed, A., \& McIntyre, R. S. (2020). Impact of COVID-19 Pandemic on Mental Health in the General Population: A Systematic Review. Journal of Affective Disorders, 277, 55-64. https://doi.org/10.1016/j.jad.2020.08.001

Zhang, E. J., Aitchison, L. P., Phillips, N., Shaban, R. Z., \& Kam, A. W. (2021). Protecting the Environment from Plastic PPE. BMJ, 372, n109. https://doi.org/10.1136/bmj.n109

Zhang, S. X., Liu, J., Jahanshahi, A. A., Nawaser, K., Yousefi, A., Li, J., \& Sun, S. (2020). At the Height of the Storm: Healthcare Staff's Health Conditions and Job Satisfaction and Their Associated Predictors during the Epidemic Peak of COVID-19. Brain, Behavior, and Immunity, 87, 144-146. https://doi.org/10.1016/j.bbi.2020.05.010 